\title{
Formulación y Uso de las Preguntas en la Clase de Ciencias Naturales a Partir de las Creencias de los Profesores. Un Estudio en la Región Metropolitana de Santiago, Chile
}

\author{
Carol L. Joglar(1), Sandra P. Rojas-Rojas ${ }^{(2)}$ y Miguel A. Manzanilla(3) \\ (1) Universidad de Santiago de Chile, Facultad de Química y Biología, Departamento de Biología, Santiago, Chile. \\ (e-mail: carol.joglar@usach.cl) \\ (2) Universidad de Santiago de Chile, Facultad de Química y Biología, Departamento de Ciencias del Ambiente, \\ Santiago, Chile. (e-mail: sandra.rojas.r@usach.cl) \\ (3) Universidad de Los Andes, Facultad de Humanidades y Educación. Departamento de Medición y Evaluación, Mérida \\ Venezuela. (e-mail: miguel86manza@gmail.com)
}

Recibido Dic. 21, 2018; Aceptado Mar. 5, 2019; Versión final Jun. 26, 2019, Publicado Oct. 2019

\begin{abstract}
Resumen
Esta investigación tuvo como objetivo identificar las creencias del profesorado en ciencias naturales de la enseñanza media en una región de Chile acerca de la formulación y el uso de las preguntas en sus clases. Se aplicó un cuestionario tipo Likert con 120 ítems, a 184 profesores de Química, Física, Biología y Ciencias Naturales de 92 colegios de la Región Metropolitana de Santiago, Chile. Para depurar la cantidad de ítems se realizó un análisis de correspondencia múltiple en dos momentos, tomando en cuenta el tipo de variable ordinal y el nivel de aporte informacional de cada uno, obteniendo 35 ítems en total. Los resultados indican tres grupos de creencias de los profesores: tradicional en avance, en tránsito y catalizador. Aunque al optar por creencias más constructivistas acerca de la formulación y uso de las preguntas, estas creencias se caracterizan por una tendencia a un cambio de paradigma, aunque ello no indica que esto se aplicará en las clases de ciencias en el corto plazo.
\end{abstract}

Palabras clave: preguntas; reflexión docente; creencias; enseñanza de las ciencias.

\section{Formulation and use of the Questions in the Class of Science Based on the Beliefs of Teachers. A Study in the Metropolitan Region of Santiago, Chile}

\begin{abstract}
This study aimed at identifying the beliefs of high school science teachers in a region of Chile, regarding the formulation and use of questions in their classrooms. A Likert-type questionnaire of 120 items was applied to 184 chemistry, physics, biology and natural sciences teachers from 92 high schools in the Santiago Metropolitan Region, Chile. In order to refine the questionnaire items, a two-session multiple correspondence analysis was carried out, taking into account the type of ordinal variable and the level of informational contribution of each item, obtaining a total of 35 . The results indicate three groups of beliefs in teachers: traditional in progress, in transit, and catalyst. Although, by changing to a more constructivist belief about the formulation and use of questions, these beliefs are characterized by a tendency to a change of paradigm, although this does not indicate that this will be applied in science classes in a short time.
\end{abstract}




\section{INTRODUCCIÓN}

En el ámbito científico, el cuestionamiento hacia una teoría es común y en general, emerge desde tensiones cognitivas y de la inconformidad generada a partir de explicaciones dominantes. En este contexto las preguntas son el motor de propulsión del conocimiento, su avance y eje central del quehacer del científico, llegando a afirmar que sin preguntas no puede haber conocimiento científico (Bacherlard, 2000). Por consiguiente, ellas aportan al surgimiento de nuevas propuestas y maneras de explicar los fenómenos (Márquez y Roca, 2006). Al considerar relevante el rol de las preguntas para el desarrollo de la investigación científica (Graesser et al., 2005), no es posible obviar su papel en la enseñanza-aprendizaje de la ciencia escolar, donde adquieren protagonismo y pueden ser más potentes que las propias respuestas (Márquez y Roca, 2006), de ahí que se le reconozca su papel central en estos procesos, puesto que son precisamente las que, en la mayoría de las ocasiones, potencian o dificultan las oportunidades de acceso al contenido científico y su comprobación de dominio (Ernst-Slavit y Pratt, 2017), al permitir que el estudiante encuentre su propio "camino cognitivo" (Chin, 2007) lo que posibilita la construcción y comprensión personal de las nociones científicas que se deseen enseñar (Lee, 1999), asimismo, logra la activación de conocimientos previos y la negociación del significado en aulas dialógicas centradas en el estudiante al facilitar las interacciones entre estudiantes y profesores (Nawani, et al., 2018). Por lo tanto, el saber formular buenas preguntas está en el centro del pensamiento científico y su desarrollo como es el caso de la argumentación científica (Osborne, 2012), la modelización (Monteiro, et al., 2009) y la indagación (Couso, 2014).

En los estudios que se refieren al sujeto que pregunta o es cuestionado, las preguntas fomentan la reflexión (Wertsch, 1988), favorecen la metacognición auto inducida y el desarrollo independiente del pensamiento de otros, además permiten que este proponga nuevas estrategias o nuevas maneras de entender situaciones que están consolidadas desde un paradigma dominante. El auto preguntarse es propulsor de la manera de pensar del estudiante, por ello, su fomento promueve en quien aprende a preguntar, el desarrollo del pensamiento complejo. Por ende, en el aula de ciencias, las preguntas apuntan a desarrollar un sujeto que no depende del pensamiento de su profesor o de sus colegas, por lo que es muy difícil que vuelva a mirar las situaciones comunes de forma convergente, lo que es relevante en el aprendizaje y desarrollo de las ciencias. Las investigaciones acerca de las preguntas en la educación científica han sido un área de bastante interés por varias décadas, especialmente las últimas tres, lo que demuestra una preocupación constante en este campo. Además de ser analizadas en el discurso de aula desde su función e interacción comunicativa (Lemke, 1990; Mortimer y Scott, 2003; Chin, 2007; De Longhi, et al., 2012; Kelly, 2014; Cutrera y Stipcich, 2016) se han centrado en el discurso del profesor (Machado y Sasseron, 2012). Otros estudios en cambio, se han enfocado en comprender como las preguntas son construidas cuando se evalúan contenidos en formatos escritos en el aula (Sanmartí y Marchán, 2014) y cómo estas se presentan al estudiantado en los textos escolares (Roca y Márquez, 2005; Dávila y Talanquer, 2009; Nakiboğlu y Yildirir, 2011; Pappa y Tsaparlis, 2011) o en analizar el efecto de las preguntas investigables en diferentes ciclos formativos de enseñanza y su promoción como competencia de pensamiento científico (Sanmartí y Márquez, 2012; Ferrés, 2017; Rojas y Joglar, 2019) y más recientemente: a) tipo de preguntas formuladas por los estudiantes (Chin, 2001; Chin, 2002; Mazzitelli, et al., 2009; Roca, et al., 2013); b) tipo de preguntas durante actividades y prácticas de investigación científica en las que se promocionó la participación de estudiantes y la comprensión del lenguaje científico (Benedict-Chambers, et al., 2017); c) análisis de preguntas realizadas por el profesor según género e interacciones de clase (Eliasson, et al., 2016) y d) análisis de las respuestas de los niños y niñas cuando un profesor o profesora pregunta (Eliasson, et al., 2017).

Cada profesor tiene una trayectoria única y está mediada por sus creencias (Contreras, 2008) y prioridades (Forbes y Davis, 2010) por lo tanto su impacto sobre el modelo de enseñanza y su accionar en el aula es un tema con varias líneas de investigación, las cuales han contribuido a comprender el conocimiento docente e indirectamente, a comprender su efecto sobre el aprendizaje del estudiantado, replanteando el entendimiento de las teorías sobre el conocimiento profesional del profesorado de ciencias, al posibilitar su comprensión y desarrollo, lo que puede ser un valioso constructo psicológico. Por lo tanto, investigar un tipo de creencia, puede ser de gran utilidad para la educación, pues es un proceso en el cual su verbalización puede posibilitar la toma de conciencia y la formalización del pensamiento (Martín, 2009). Aunque el avance en estudios relacionados con el tema de las preguntas ha sido significativo, en los últimos años, algunos estudios han abordado las creencias de los profesores de Ciencias relacionados especialmente con la enseñanza de las ciencias en la educación primaria (Nawani, et. al., 2018; Forbes y Davis, 2010). En la Ciencia Escolar, la pregunta puede ser un promotor o un limitador de la metacognición (Labarrere, 2012) y de la autorregulación de los aprendizajes del estudiantado (Chin y Osborne, 2010), donde el tipo de pregunta planteada por el docente determinará el proceso mental que el estudiante deberá desarrollar para su respuesta e indicará a este, los límites de libertad que tendrá para desarrollar (Colás, 1983), por tanto adquiere una mayor relevancia el estudiar la competencia que tiene el profesorado para formular preguntas, ya que existe una relación entre la competencia del profesorado y de sus estudiantes (Redfield y Rousseau, 1981). A pesar de que su uso tiene ventajas, su formulación puede ser una tarea muy compleja para los profesores (Graesser et. al., 2005; 
Chin, 2007; Nawani, et. al. (2018). Si bien se tiene claro que la mejora de la calidad de las preguntas en el aula no es la única manera de transformar la enseñanza de las ciencias, debido al rol que ellas ocupan en la generación del pensamiento científico, es relevante problematizar la formulación y el uso de las mismas en espacios de formación profesional (Joglar y Rojas, 2019). Las preguntas usadas en el aula durante la interacción, generan ambientes colaborativos y de reconstrucción social (Wertsch, 1988), al ser "andamios de ideas" le permiten repensar, desde nuevos enfoques, estrategias y maneras de entender situaciones consolidadas en paradigmas dominantes, es decir, que las preguntas adquieren un papel significativo al retirar "Io normal". Por consiguiente, entendemos que formular preguntas en la clase de ciencias es una competencia de pensamiento científico (Joglar, 2015) de profesores y estudiantes, que considera procesos cognitivos superiores, lingüísticos, contextuales y emocionalmente complejos, que deberían ser tenidos en cuenta en el diseño de actividades de enseñanza de las ciencias naturales. Por lo tanto, es una competencia metacomprensiva que necesita ser enseñada, promovida y desarrollada, no se genera de manera espontánea, por ello, el profesor debe aprenderla, enseñarla y desarrollarla durante su formación inicial o continua (Silvestri, 2006).

Estudios internacionales como la prueba PISA del año 2015 indican que cuatro de cada diez estudiantes chilenos no tienen las habilidades científicas mínimas en lo que se refiere a la resolución de problemas (OCDE, 2016). Esto cobra sentido cuando contrastamos los resultados de la investigación de Malvaez, Joglar y Quintanilla (2013), donde se identifica que el uso dado a las preguntas en ciencias por parte de los estudiantes en Chile es, predominantemente, para la búsqueda de información, es decir, que falta una cultura indagatoria de calidad al interior de las salas de clases en nuestro país. Además estos resultados, de cierto modo, son coherentes con otras investigaciones relacionadas al tipo de preguntas que realizan los profesores. Estos estudios reportan que el $60 \%$ de las preguntas realizadas por los docentes en las aulas de ciencias se refieren a la memorización de hechos, el $20 \%$ requieren que los estudiantes piensen y el $20 \%$ restante, son de procedimientos (Blosser, 2000), lo que demuestra, que todavía los profesores enseñan desde marcos positivistas en los cuales la memorización del contenido tiene mayor relevancia que el desarrollo del pensamiento científico (Porlán et al., 2011). Las preguntas formuladas a partir de un enfoque tradicional presentan como características: el uso centrado en la evaluación, dominio del docente en la estructura discursiva, siguen una planificación rígida, favorecen la memorización, reacción positiva a una respuesta "correcta" y evaden las respuestas equivocadas, además, es el profesor quién valida el conocimiento. Por su parte, las preguntas formuladas a partir de un enfoque constructivista, se usan para promover la exposición de ideas, el pensamiento científico con estructuras discursivas en cadena, fomentan la participación del estudiantado, promueven el pensamiento científico, la responsabilidad de generar soluciones es del estudiantado, demora para juzgar una respuesta, la validación del conocimiento es grupal y reconoce la contribución del grupo de trabajo (Chin, 2007).

Según Silvestri (2006) la habilidad para formular preguntas es de gran importancia para el adecuado proceso de aprender a comprender, para lo cual se demanda conocimiento y reflexión sobre el mismo y requiere la necesidad de estudiar el pensamiento docente, como un mecanismo para comprender su enseñanza, sus decisiones didácticas y sus creencias. Las creencias del profesorado acerca de cómo enseñan y aprenden los estudiantes, son verdades personales, están directamente relacionadas con sus experiencias, logros, vínculos con la acción en el aula, sus decisiones didácticas y el discurso (Pajares, 1992; Bryan, 2003). Tienen como características la subjetividad, la perseverancia, responden a experiencias reiteradas, se adquieren de manera no reflexiva, se transmiten culturalmente, en general, provienen del sentido común. Por ende, su estudio adquiere un valioso constructo psicológico para la formación del profesorado. Investigar un tipo de creencia posibilita una exploración viable y de gran utilidad para la educación, proceso en el cual la verbalización puede posibilitar la toma de conciencia y la formalización del pensamiento (Pajares, 1992; Martín, 2009).

Si se considera a las preguntas como uno de los recursos usados con mayor frecuencia por los docentes durante la enseñanza y con mayor impacto en su aprendizaje, es preocupante los resultados de la evaluación docente en Chile sobre este tema. Estos señalan que ocho de cada diez profesores, se clasificaron en niveles básicos e insuficientes en cuanto a la calidad de sus preguntas en el aula (Sun, et al., 2011). Por tanto, urge la necesidad de formar al profesorado en la formulación de las preguntas, así como de asignar un buen uso en clases de ciencias.

Desde las políticas públicas para la enseñanza de las ciencias en Chile, se ha priorizado un enfoque hacia la enseñanza por la indagación (Cofré, et al., 2010), en donde la formulación de preguntas es una habilidad básica. Sin embargo, es necesario que estas propuestas tomen en cuenta las creencias que tienen los profesores acerca de las mismas y su uso en el aula, ya que estas responden a experiencias reiteradas, adquiridas de manera no reflexiva, naturalizadas desde el sentido común, con ausencia de experiencias de crítica, pero si, utilizadas en la acción docente. Por lo tanto, comprender el accionar del profesorado desde sus creencias, podrá generar explicaciones frente a indicadores que las describan y en consecuencia comprender cómo inciden en su toma de decisiones. 
La trascendencia del tema y la carencia de estudios de esta naturaleza en Chile, permiten que sea relevante indagar acerca de qué creen los profesores sobre la formulación y uso de las preguntas en las clases de ciencias. Con esta intención, se diseñó un cuestionario a profesores de las áreas de Ciencias Experimentales que trabajan en Santiago de Chile, con el propósito de analizar en profundidad: ¿Cuáles son las creencias de la muestra en cuanto a la formulación de preguntas en la clase de ciencias?, ¿Qué elementos de las dimensiones (¿qué?, ¿cómo? y ¿por qué?) los profesores consideran en la formulación de preguntas?, ¿Cuáles son las creencias de la muestra en cuanto al uso de las preguntas?, ¿Qué elementos de las dimensiones (¿Cuándo pregunto?, ¿A quién pregunto?, ¿Para qué pregunto?, ¿Cómo me siento cuando pregunto?, ¿Cómo se sienten mis estudiantes cuando pregunto?) los profesores incluyen acerca del uso que dan a las preguntas?.

\section{METODOLOGÍA}

Esta investigación relata los resultados de la primera etapa de un proyecto de investigación en el cual se diseñó, validó y aplicó un cuestionario para identificar las creencias de profesores de ciencias acerca de la formulación y uso de las preguntas en el aula.

A partir de la Tabla 1 de distribución de frecuencias, se identificó una muestra conformada por 184 profesores de ciencias (Biología, Química, Física y Ciencias Naturales) que trabajan en 92 colegios de tipo científico humanista o liceos técnicos con dependencia administrativa municipal, particular subvencionado y particular pagado de la Región Metropolitana de Chile. Los profesores ejercen y desarrollan sus actividades de docencia en enseñanza de las ciencias, en nivel básico y/o medio y aceptaron voluntariamente participar de la investigación a través de la firma del consentimiento informado.

Tabla 1: Descriptivos principales de la muestra

\begin{tabular}{|c|c|c|c|c|c|c|c|c|c|c|c|c|c|c|c|c|}
\hline \multicolumn{3}{|l|}{ Variable } & \multicolumn{3}{|l|}{ Edad } & \multicolumn{2}{|c|}{ Género } & \multicolumn{4}{|c|}{ Años de experiencia } & \multicolumn{5}{|c|}{ Dep. Administrativa } \\
\hline Categ. & $<30$ & $30 / 39$ & $40 / 49$ & $50 / 59$ & $60>$ & $\mathrm{H}$ & M & $<10$ & $11 / 19$ & $20 / 29$ & $30>$ & M & $\mathrm{M} / \mathrm{P}$ & $\mathrm{P}$ & PS & NE \\
\hline Frec. & 66 & 55 & 31 & 20 & 12 & 69 & 115 & 109 & 36 & 17 & 22 & 29 & $2^{*}$ & 35 & 50 & 68 \\
\hline$\%$ & 36 & 30 & 17 & 11 & 7 & 37 & 63 & 59 & 20 & 9 & 12 & 16 & $1^{*}$ & 19 & 27 & 37 \\
\hline
\end{tabular}

Respecto de la edad de los profesores, el $66 \%$ son menores de 40 años; el $63 \%$ de los participantes son mujeres; el 59\% de la muestra tiene menos de 10 años de experiencia docente y trabajan mayormente en colegios con dependencia administrativa particular y particular subvencionados (19\% y $27 \%$ ).

\section{El cuestionario}

Inicialmente se construyeron 121 afirmaciones sobre las preguntas, siendo 66 de ellas con un enfoque tradicional y 55 con un enfoque constructivista, categorizadas en ocho dimensiones y que se reúnen en 2 grupos: 1) Formulación (¿Cómo pregunto?,¿Qué pregunto?, ¿Por qué pregunto?) y 2) El uso (¿Cuándo pregunto?, ¿A quién pregunto?, ¿Para qué pregunto?, ¿Cómo me siento cuando pregunto?, ¿Cómo se sienten mis estudiantes cuando pregunto?). Este cuestionario se construyó como una escala psicométrica tipo Likert, la cual buscó en cada afirmación, identificar el grado de acuerdo con cada una de las afirmaciones: totalmente de acuerdo (TA), parcialmente de acuerdo (PA), parcialmente en desacuerdo (PD) y totalmente en desacuerdo (TD). Cada afirmación buscaba identificar las creencias de los profesores desde los enfoques "tradicional" o "constructivista" al seguir la propuesta adaptada de Chin (2007).

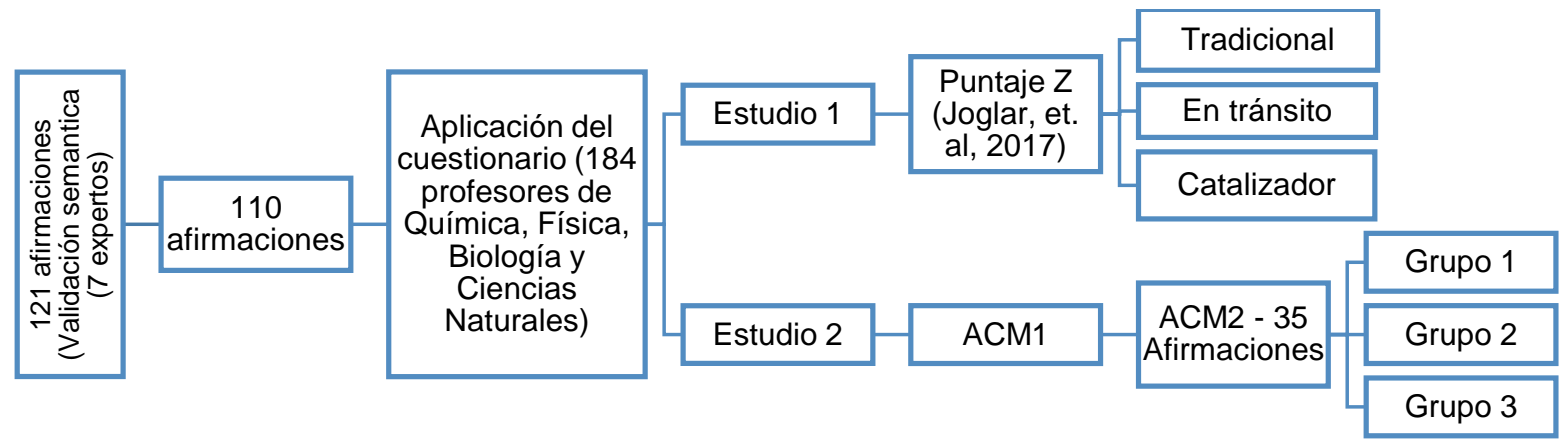

Fig. 1: proceso de validación del cuestionario

La validación de los constructos del cuestionario fue de tipo semántica (Fig. 1) a través del juicio de 7 expertos, doctores en educación o didáctica de las ciencias. Cada ítem fue juzgado a partir de: a) no cumple con los criterios evaluados, b) cumple con la relevancia, sin embargo requiere modificaciones y c) cumple todos los 
aspectos. El cuestionario final quedó compuesto por 110 afirmaciones. Los resultados preliminares de la aplicación del cuestionario fueron presentados en Joglar, Navarro y Rojas (2017) en el que a través del puntaje $Z$ se pudo identificar 3 grupos de respuestas: sujetos que estaban de acuerdo con afirmaciones con enfoque "tradicional en avance" (36,6\%), aquellos que estaban de acuerdo con afirmaciones con enfoque constructivista (38.4\%) también llamados "catalizadores" y $25 \%$ de la muestra restante que presentó creencias mixtas, llamadas "en tránsito" (Fig. 1). Sin embargo, la gran cantidad de constructos del cuestionario final (110) analizados en el estudio 1, no permitió obtener una caracterización detallada del perfil y tampoco relacionarlo con las dimensiones del cuestionario anteriormente mencionadas, en consecuencia, no se pudo establecer claramente aquellas creencias relacionadas con la formulación y aquellas relacionadas con el uso de las preguntas. Esto nos llevó a realizar el estudio 2 (Fig. 1), donde se usó el Análisis de Correspondencia Múltiple $(\mathrm{ACM})$, el cual es un índice de discriminación apropiado para estudios con variables categóricas u ordinales, como es el caso de las respuestas en cuestionarios tipo Likert. A través de este análisis fue posible reducir el número de afirmaciones e identificar las creencias propuestas en la investigación.

\section{Diseño de la investigación}

Las etapas que componen el diseño de la investigación son: a. ACM1 - reducción de las afirmaciones. b. AMC2 - determinar los grupos que contienen las afirmaciones que mejor discriminan las características del perfil y c. perfil de las creencias

\section{Reducción de las afirmaciones}

Análisis de correspondencia múltiple 1 (ACM1). En este análisis (Abascal y Grande, 2017) se evaluaron las variables transformadas con sus respectivas medidas de discriminación a través de representaciones gráficas, las cuáles fueron, afirmaciones con el mayor nivel de explicabilidad de la variabilidad total que permite caracterizar el perfil de creencias acerca de la formulación y el uso de las preguntas. Para la reducción de las afirmaciones se realizó el ACM1 (SPSS v.23) donde se conformó una nube de puntos en dos dimensiones, observando las posiciones relativas de estos, pero manteniendo el máximo de posiciones relativas en la nube original (figura 2).

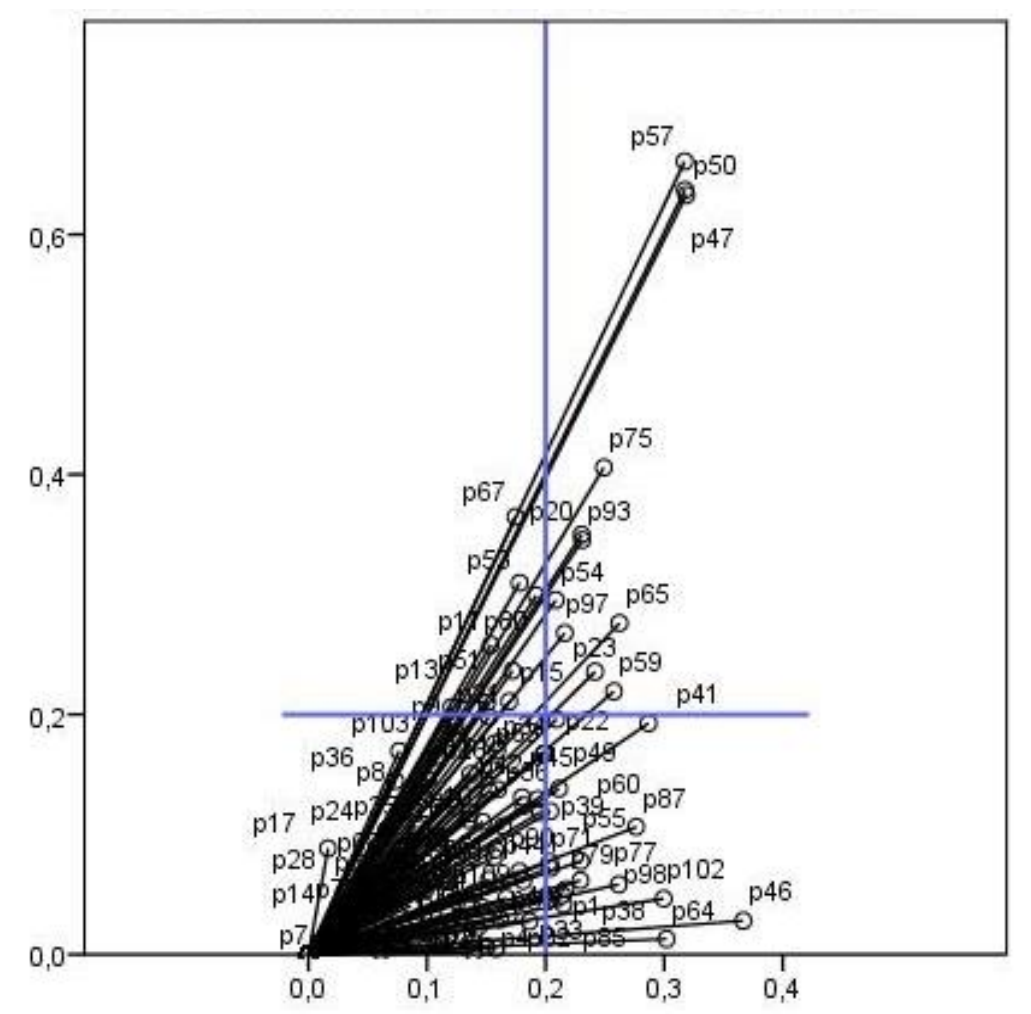

Fig. 2: Medidas discriminantes entre las afirmaciones y normalización de las variables con respecto al ACM 1.

Al revisar este conjunto de medidas fue posible ubicar afirmaciones que no aportaron suficiente variabilidad a cada eje cartesiano, por lo tanto se decide descartar las afirmaciones que presentaron una discriminación por debajo del $20 \%$ y el grado de asociación entre ellas, lo que se puede observar en la figura 2 como un punto de corte (azul) en ambos ejes. En consecuencia, se descartan 75 ítems del cuestionario inicial, quedando reducido a 35 afirmaciones relevantes (Tabla 2). 
Tabla 2. Afirmaciones y enfoque en cada dimensión en la formulación y el uso de las preguntas en el aula de ciencias.

\begin{tabular}{|c|c|c|c|}
\hline \multirow{12}{*}{ 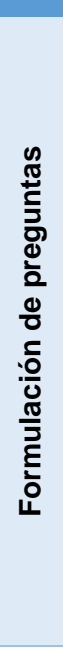 } & Dimensión & No/Enfoq & Afirmación \\
\hline & \multirow{5}{*}{$\begin{array}{l}\text { ¿Cómo } \\
\text { pregunta? }\end{array}$} & $54-\mathrm{C}$ & Una buena pregunta en clases de ciencias puede tener más de una posible respuesta. \\
\hline & & $64-\mathrm{T}$ & $\begin{array}{l}\text { Al diseñar una pregunta para mis clases, me cercioro de que su respuesta sea breve y } \\
\text { concisa. }\end{array}$ \\
\hline & & $75-C$ & $\begin{array}{l}\text { Las preguntas abiertas que realizo en la clase me permiten conocer cómo piensan mis } \\
\text { estudiantes }\end{array}$ \\
\hline & & $79-\mathrm{T}$ & $\begin{array}{l}\text { Tengo dificultades para usar preguntas abiertas en las evaluaciones sumativa, porque son } \\
\text { difíciles de evaluar de manera objetiva el contenido aprendido. }\end{array}$ \\
\hline & & $85-\mathrm{T}$ & Prefiero preguntas cerradas porque llevan directo al tema que necesito que piensen. \\
\hline & \multirow[t]{4}{*}{$\begin{array}{l}\text { ¿Qué } \\
\text { pregunta? }\end{array}$} & $39-\mathrm{C}$ & $\begin{array}{l}\text { Hacer preguntas acerca de un contenido que todavía no he enseñado, orienta el } \\
\text { aprendizaje de mi estudiante. }\end{array}$ \\
\hline & & $46-\mathrm{T}$ & $\begin{array}{l}\text { Hacer preguntas sobre un contenido que todavía no he enseñado confunde a los } \\
\text { estudiantes durante su aprendizaje. }\end{array}$ \\
\hline & & $102-\mathrm{T}$ & $\begin{array}{l}\text { Considero que no es adecuado preguntar acerca de temas involucren opiniones acerca de } \\
\text { dilemas humanos en la clase de ciencia (p.e. aborto) }\end{array}$ \\
\hline & & $110-\mathrm{T}$ & No considero relevante preguntar en mis clases temáticas que involucren el colegio. \\
\hline & \multirow{2}{*}{$\begin{array}{l}\text { ¿Por qué } \\
\text { pregunta? }\end{array}$} & $47-C$ & Una pregunta debe ser mediadora entre lo que el estudiante sabe y lo que desea aprender. \\
\hline & & $53-\mathrm{C}$ & $\begin{array}{l}\text { Considero importante los momentos que mis estudiantes cuestionan lo que estoy } \\
\text { enseñando. }\end{array}$ \\
\hline \multirow{24}{*}{ 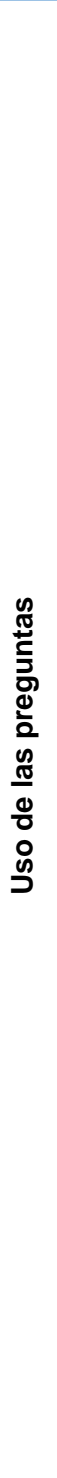 } & \multirow[t]{5}{*}{$\begin{array}{l}\text { ¿Cuándo } \\
\text { pregunta? }\end{array}$} & $20-C$ & $\begin{array}{l}\text { Es útil preguntar al estudiantado lo que piensan que han aprendido al final de las clases } \\
\text { de ciencias }\end{array}$ \\
\hline & & $57-C$ & $\begin{array}{l}\text { Considero eficaz el trabajo grupal cuando el estudiantado necesita resolver una guía de } \\
\text { preguntas abiertas }\end{array}$ \\
\hline & & $59-\mathrm{T}$ & $\begin{array}{l}\text { No considero importante preguntar al estudiantado lo que piensan que han aprendido al fin } \\
\text { de una clase de ciencias. }\end{array}$ \\
\hline & & $77-\mathrm{T}$ & $\begin{array}{l}\text { Solamente las preguntas realizadas en actividades prácticas tienen sentido para } \\
\text { desarrollar el pensamiento científico. }\end{array}$ \\
\hline & & $86-C$ & $\begin{array}{l}\text { Es importante partir la clase con una pregunta que problematice lo enseñado en la clase } \\
\text { anterior. }\end{array}$ \\
\hline & \multirow[t]{14}{*}{ ¿Para qué? } & $11-\mathrm{T}$ & $\begin{array}{l}\text { Considero que, en las clases de ciencias, una pregunta divergente confunde al } \\
\text { estudiantado. }\end{array}$ \\
\hline & & $13-\mathrm{T}$ & $\begin{array}{l}\text { Me gusta usar mis preguntas como un mecanismo de guía del pensamiento del estudiante, } \\
\text { llevándolo a centrarse en el contenido que estamos trabajando. }\end{array}$ \\
\hline & & $15-C$ & $\begin{array}{l}\text { Preguntar en clases de ciencias permite que el estudiantado se articule con temas } \\
\text { adicionales a los que enseño. }\end{array}$ \\
\hline & & $22-C$ & Usar preguntas en clases puede mejorar el interés de mis estudiantes hacia la ciencia. \\
\hline & & $23-C$ & $\begin{array}{l}\text { Las preguntas en la clase de ciencias deben promover el pensamiento divergente del } \\
\text { estudiantado. }\end{array}$ \\
\hline & & $49-C$ & $\begin{array}{l}\text { Una manera de ayudar al estudiante a identificar lo que quiere preguntar es pidiéndole que } \\
\text { reformule su pregunta. }\end{array}$ \\
\hline & & $50-\mathrm{T}$ & Preguntar en la clase de ciencias distrae al estudiantado. \\
\hline & & $51-\mathrm{C}$ & $\begin{array}{l}\text { Cuando realizo una pregunta a mis estudiantes deseo que respondan lo que realmente } \\
\text { saben. }\end{array}$ \\
\hline & & $60-\mathrm{C}$ & Una pregunta del estudiante me permite identificar lo que no ha aprendido. \\
\hline & & $65-\mathrm{T}$ & $\begin{array}{l}\text { No considero importante la redacción que utilizan los estudiantes en sus respuestas a } \\
\text { preguntas de desarrollo. }\end{array}$ \\
\hline & & $80-C$ & $\begin{array}{l}\text { Las preguntas abiertas que realizo en la clase me permiten conocer cómo piensan mis } \\
\text { estudiantes. }\end{array}$ \\
\hline & & $93-\mathrm{C}$ & $\begin{array}{l}\text { Me gusta aprovechar las respuestas incorrectas de los estudiantes como un momento de } \\
\text { aprendizaje en las clases de ciencias. }\end{array}$ \\
\hline & & $97-\mathrm{T}$ & Una pregunta permite al estudiante identificar lo que sabe. \\
\hline & & $98-\mathrm{T}$ & $\begin{array}{l}\text { Una manera de ayudar al estudiante a identificar lo que quiere preguntar es reformulando } \\
\text { su pregunta. }\end{array}$ \\
\hline & ¿A quién? & $67-C$ & $\begin{array}{l}\text { Cada estudiante tiene formas de expresión personales, por lo tanto, deben responder de } \\
\text { manera diferente a sus colegas una misma pregunta }\end{array}$ \\
\hline & \multirow{2}{*}{$\begin{array}{l}\text { ¿Cómo se } \\
\text { siente el } \\
\text { profesor? }\end{array}$} & $38-\mathrm{T}$ & Me preocupa que mis estudiantes se den cuenta que no se la respuesta a una pregunta. \\
\hline & & $87-\mathrm{T}$ & Cuando los estudiantes me hacen preguntas inesperadas, me siento nervioso(a) \\
\hline & \multirow{2}{*}{$\begin{array}{l}\text { ¿Cómo se } \\
\text { sienten sus } \\
\text { estudiantes? }\end{array}$} & $1-\mathrm{T}$ & Mis estudiantes suelen molestarse si respondo sus preguntas con otras preguntas. \\
\hline & & $41-\mathrm{T}$ & $\begin{array}{l}\text { Cuando el estudiante responde una pregunta de manera compleja, es porque está } \\
\text { intentando solapar una falta de conocimiento. }\end{array}$ \\
\hline
\end{tabular}

Análisis de Correspondencia Múltiple 2 (ACM2). Una vez reestructurada la base de datos según los resultados a partir del ACM1, se procedió a replicar el análisis con las 35 afirmaciones restantes, obteniendo medidas discriminantes con niveles de variabilidad explicada, superior al $20 \%$, en al menos uno de los ejes cartesianos, identificando, con mayor claridad, las tendencias o patrones de respuesta y correlación (Fig. 3). 


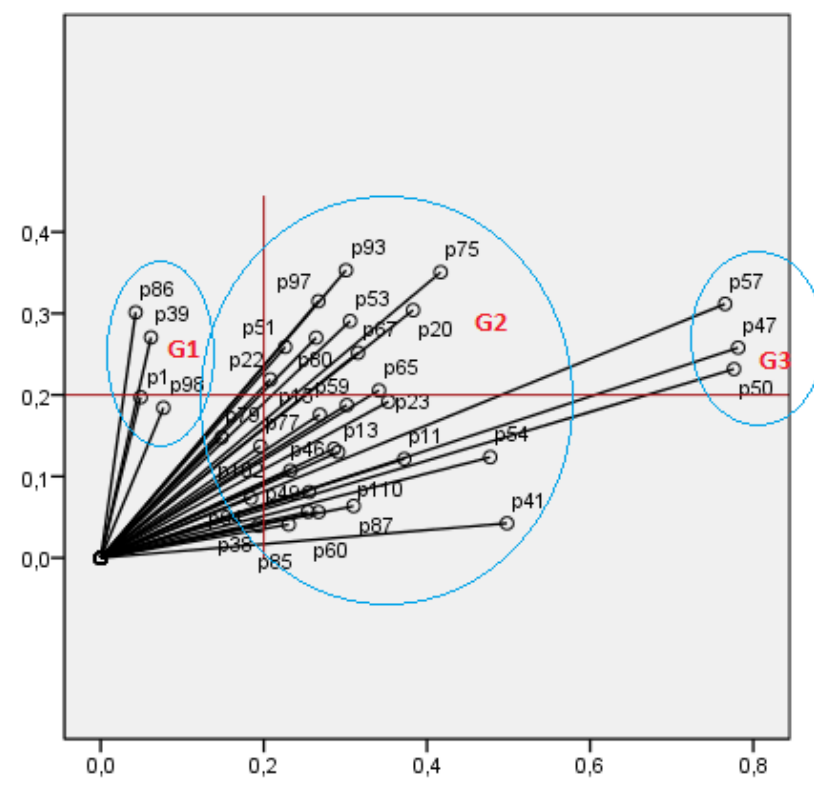

Fig. 3. Medidas discriminantes entre las afirmaciones y normalización de las variables con respecto al ACM 2

En la figura 3 se puede observar de izquierda a derecha, como se agrupan las afirmaciones que conforman al instrumento definitivo (35 ítems), en función de las respuestas dadas por la muestra, reflejando en primera instancia una evidente oposición entre el grupo 1 y el grupo 3 (G1 y G3) y el grupo 2 (G2) que contiene una proporción considerable de atributos mixtos observados, y que buscamos analizar con mayor profundidad para caracterizar el perfil del profesorado de ciencias en relación a sus creencias acerca de la formulación y uso de las preguntas en el aula. Es importante tener en cuenta que luego del ACM2, el Alfa de Cronbach para el instrumento final fue de 0.87 , lo que confirma un alto nivel de confiabilidad.

\section{RESULTADOS}

Para caracterizar el perfil de creencias a partir del análisis de las respuestas del cuestionario, se siguieron 4 pasos que detallaremos a continuación: 1) se agruparon las afirmaciones existentes en los grupos arrojados en el ACM2 ,2) se caracterizó el comportamiento de las respuestas de la muestra en las afirmaciones de las dimensiones presentes en el cuestionario, 3) se agruparon las dimensiones que componen cada creencia y las respuestas de los grupos de afirmaciones en cuanto a la formulación y uso de la pregunta y 4) se describe el perfil de las afirmaciones, es decir, lo que finalmente constituyen el perfil de las creencias en "tradicional en avance", en "tránsito" y "catalizadores".

1) Agrupación de afirmaciones en cuanto a los extremos de creencias: de los tres grupos identificados a partir del ACM2, el G1 (Fig. 4) y el G3 (Fig. 5) presentan comportamientos opuestos (Fig. 3). El G1 (Fig. 4) está compuesto de dos afirmaciones cuyo enfoque es tradicional y dos afirmaciones con enfoque constructivista. El comportamiento de las respuestas dadas por la muestra a las afirmaciones constructivistas y tradicionales son predominantemente TA y PA indicando que apoyan tanto afirmaciones constructivistas y afirmaciones tradicionales.

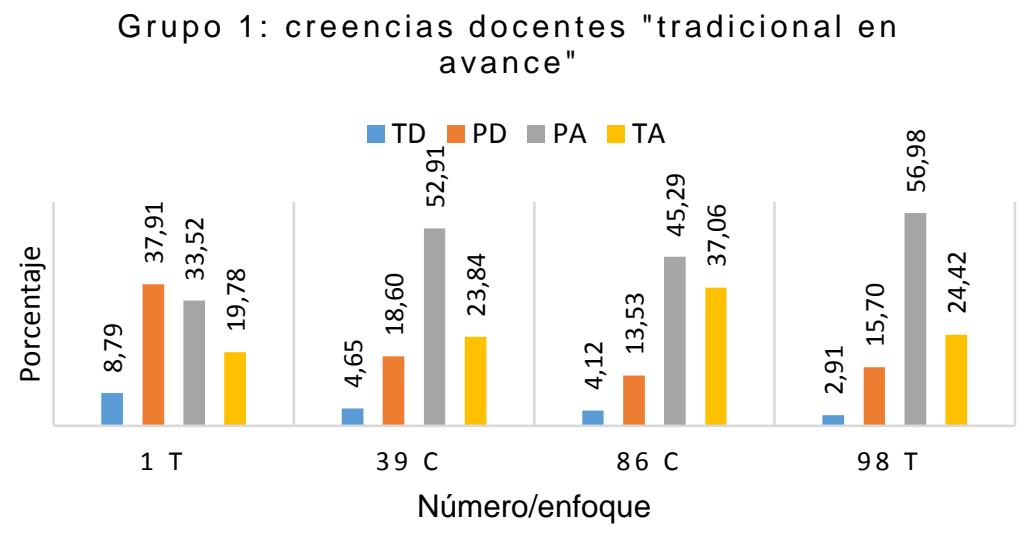

Fig. 4 Comportamiento de las respuestas a las afirmaciones que componen el grupo 1 C (enfoque constructivista del ítem) y T (enfoque tradicional del ítem). 
Esto indica que este grupo presenta un comportamiento "tradicional en avance" hacia el G2, o sea, ha sido mencionado en otras investigaciones (Vázquez-Bernal, Jiménez-Perez y Jiménez, 2016) que las creencias no son "puras" en lo tradicional, más bien se percibe un acercamiento a las creencias del G2. Esto justifica la necesidad de profundizar este estudio en el comportamiento de estas afirmaciones en las dimensiones del instrumento definidas a priori.

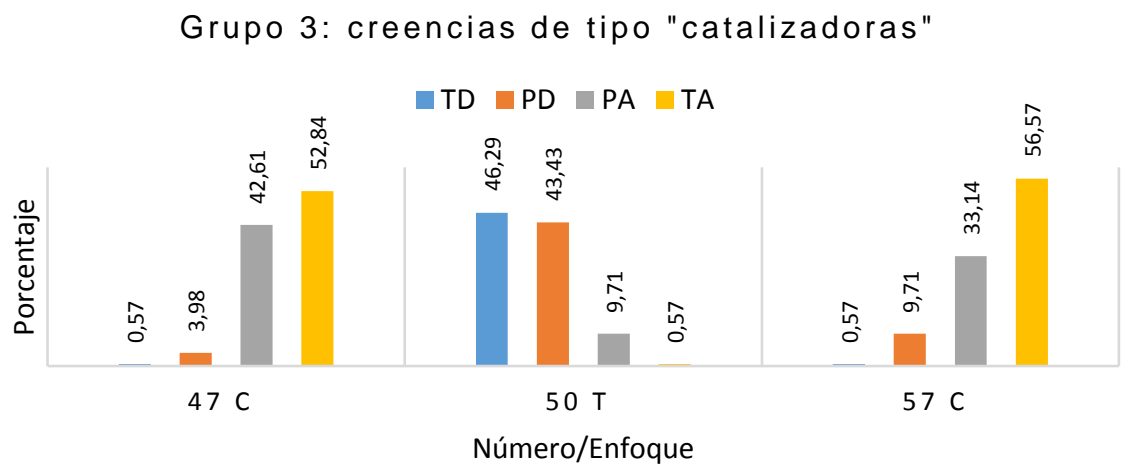

Fig. 5: Comportamiento de las respuestas a las afirmaciones que componen el grupo 3C (enfoque constructivista del ítem) y T (enfoque tradicional del ítem).

EI G3 (Fig. 5) contiene 3 afirmaciones, siendo 2 de enfoque constructivista y una de enfoque tradicional. El comportamiento de las respuestas pertenecientes a las afirmaciones constructivistas, son predominantemente TA y PA, y en la afirmación que, si bien es de enfoque tradicional, el comportamiento de la muestra tiende a un comportamiento constructivista, estando claramente en desacuerdo con la afirmación, caracterizando así al grupo con creencias docentes del tipo "catalizadores".

De los tres grupos identificados en el $\mathrm{ACM}_{2}$, el G2 es un grupo que presenta cerca del $50 \%$ de las afirmaciones constructivistas y el otro $50 \%$ con enfoque tradicional.

Grupo 2: creencias docentes "en tránsito"

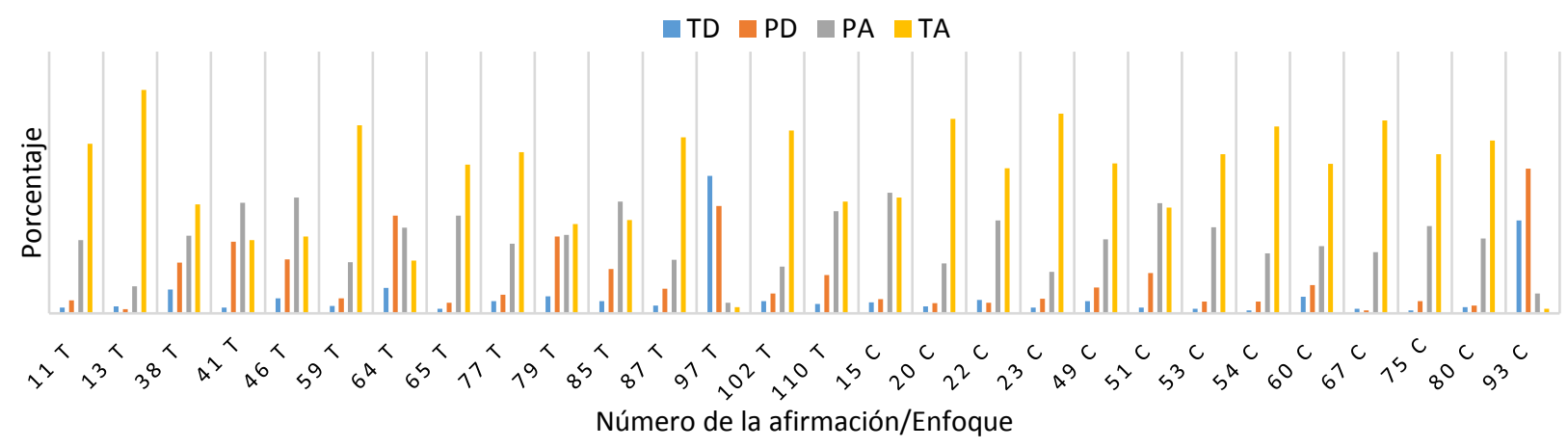

Fig. 6: Comportamiento de las respuestas a las afirmaciones que componen el grupo $2 \mathrm{C}$ (enfoque constructivista del ítem) y $\mathrm{T}$ (enfoque tradicional del ítem).

EI G2 (Fig.6) presentó 15 afirmaciones de enfoque tradicional y 13 afirmaciones de enfoque constructivista. Se observa que las afirmaciones de enfoque tradicional presentan respuestas mayormente de TA y PA con ese enfoque, excepto la afirmación 97 . En lo que se refiere a las respuestas presentadas por las afirmaciones de enfoque constructivista, presentan mayormente TA y PA con ese enfoque, excepto en la afirmación 93. Si bien la cantidad de afirmaciones no es igual, el comportamiento del grupo presenta un marcado equilibrio entre las respuestas a afirmaciones con enfoque tradicional y constructivista, indicando que las respuestas del grupo se caracterizan por creencias que están "en tránsito", como se puede verificar en la figura 3.

A partir de los resultados del agrupamiento de las afirmaciones y el comportamiento de las respuestas de la muestra, se puede entender que, si bien los tres grupos identificados con creencias docentes "tradicional en avance", "en tránsito" y "catalizador", presentan creencias opuestas que conviven en un mismo sujeto. Esto tiene coherencia con lo mencionado por Bryan (2003) y Pajares (2002) en cuanto indican la resistencia de las creencias al cambio, entendidas como verdades personales, que provienen desde el sentido común, sin pasar por reflexiones teóricas. 


\section{2) Comportamientos de las respuestas de la muestra y las dimensiones}

Tomando en cuenta los patrones descritos anteriormente, se construyeron gráficos en los cuales se buscó identificar el comportamiento de las respuestas de la muestra en las dimensiones del cuestionario. Primeramente, se mencionan las dimensiones que están agrupadas en "creencias acerca de la formulación" y a continuación, las dimensiones que componen el grupo "creencias acerca del uso de las preguntas en el aula".

\section{Formulación de preguntas}

\section{a. Dimensión ¿Cómo pregunta el profesor?}

La dimensión "¿Cómo pregunta el profesor?" contiene 5 afirmaciones, y todas pertenecen al grupo "en tránsito". De estas, 2 afirmaciones son de enfoque constructivista y tres afirmaciones de enfoque tradicional (Fig. 7). Se puede observar que las afirmaciones que tienen un enfoque constructivista presentan respuestas con un alto grado de acuerdo, sin embargo en lo que se refiere a las afirmaciones con enfoque tradicional, si bien en la afirmación 85 el comportamiento es el esperado, las respuestas hacia los ítems 64 y 79 no dejan claro un acuerdo predominante con este enfoque.

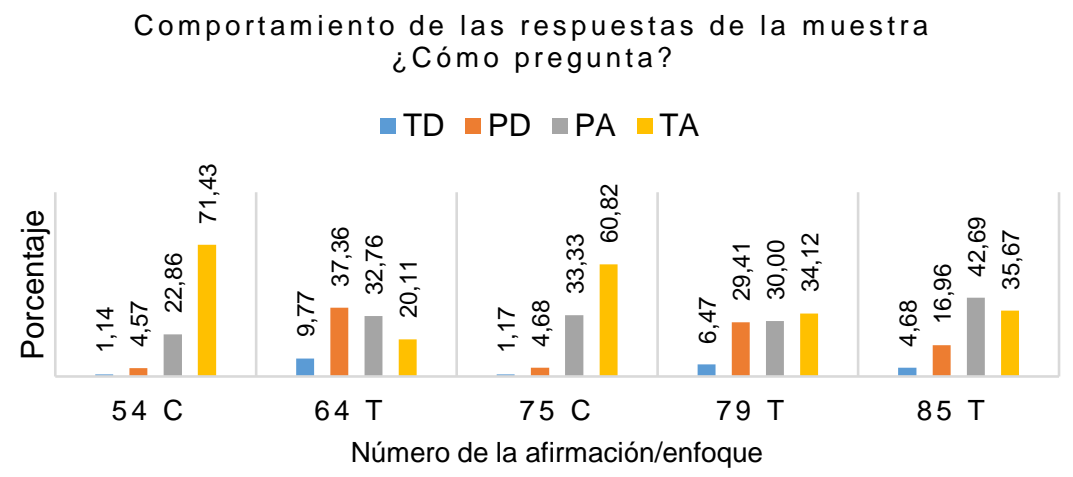

Fig. 7. Agrupación de las afirmaciones y el comportamiento de las respuestas que componen la dimensión ¿Cómo pregunta? C (enfoque constructivista del ítem) y T (enfoque tradicional del ítem).

Las respuestas de esta dimensión demuestran un enfoque constructivista cuando los profesores consideran que una buena pregunta puede tener varias respuestas posibles, y que ellas permiten conocer cómo piensan sus estudiantes. Es interesante ver este tipo de respuestas en una dimensión que busca entender como pregunta el profesorado, ya que se percibe un enfoque dogmático de la enseñanza cuando están de acuerdo en que sus preguntas tienen respuestas breves y concisas, además les cuesta usar preguntas abiertas por que es difícil evaluarlas objetivamente, a diferencia de las preguntas cerradas, las cuales direccionan el pensamiento de sus estudiantes. Estos hallazgos están de acuerdo con Nawani, et. al. (2018) donde se menciona que las preguntas de los profesores se centran en preguntas de tipo ¿qué?, ¿quién? y ¿cuándo? lo que genera una interacción monologa entre maestro y estudiante. Los mismos autores mencionan que las preguntas abiertas, también son desafiantes y posibilitan que lo enseñado sea usado para desarrollar explicaciones científicas a través de la exploración de interconexiones entre hechos, principios e ideas básicas, anclándolas a los conocimientos de la ciencia. Por el contrario, las preguntas cerradas o aquellas de niveles de pensamiento de orden inferior promueven el parafraseo del tema y su memorización.

\section{b. Dimensión ¿Qué pregunta el profesor?}

La dimensión ¿qué pregunta el profesor? contiene 4 afirmaciones, siendo una con enfoque constructivista, que también pertenece al grupo 1, y 3 afirmaciones con enfoque tradicional, que también pertenecen al grupo 2 (Fig. 8). El comportamiento de las respuestas a la afirmación constructivista y las tradicionales presenta una tendencia hacia el TA y PA, lo que era esperado.

Los profesores consideran que preguntar acerca de un contenido que todavía no han enseñado orienta el aprendizaje, sin embargo también consideran que esto confunde, lo que es contradictorio. También piensan que no es adecuado preguntar acerca de dilemas humanos que involucren temas éticos o temas relacionados con el colegio. Esta dimensión presenta respuestas contradictorias y predominan visiones dogmáticas de la naturaleza de la ciencia. Estas respuestas son preocupantes, ya que esta dimensión tiene una naturaleza epistemológica lo que tiene sentido a partir del estudio realizado por Chen, Hand, y Norton-Meier, (2017) donde se menciona que es necesario que el profesor reconsidere sus creencias acerca de las preguntas y 
que también debe tener un apoyo para su desarrollo profesional, ya que se sabe que el cambio significativo en las creencias acerca del cuestionamiento en el aula puede tomar más de 18 meses. También es interesante percibir que los profesores presentan creencias de enfoque tradicional cuando se refieren al uso de las preguntas que involucran dilemas humanos y problemáticas cercanas a los estudiantes, ya que la investigación (Eliasson, et al., 2016; Mortimer y Scott., 2003) considera que desde el sujeto que aprende, el hablar de sí mismo es fundamental para la creación de significados y el consecuente aprendizaje, donde aprender ciencias es aprender a hablar de la ciencia.

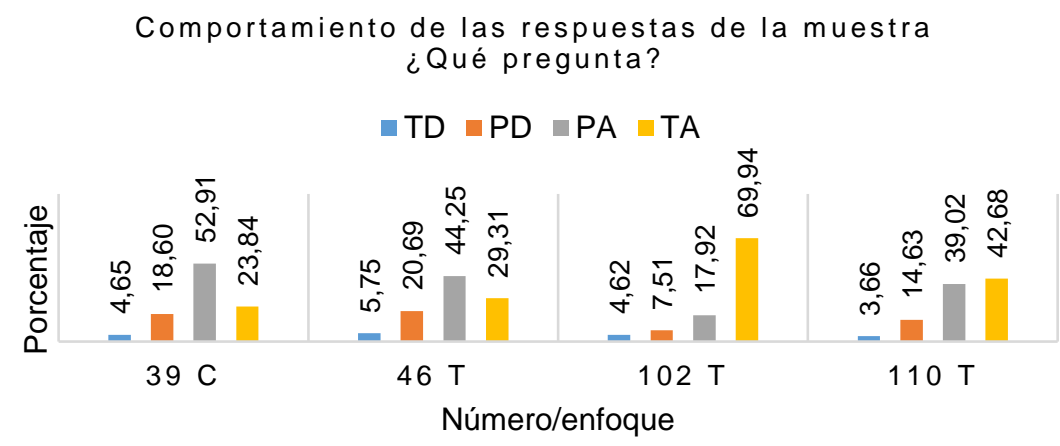

Fig. 8: Agrupación de las afirmaciones y el comportamiento de las respuestas que componen la dimensión ¿qué pregunta? C (enfoque constructivista del ítem) y T (enfoque tradicional del ítem).

c. Dimensión ¿Por qué pregunta el profesor?

La dimensión ¿Por qué pregunta el profesor? está compuesta de dos afirmaciones con enfoque constructivista, siendo que una pertenece al grupo 3 y la otra al grupo 2 (Fig. 9). El comportamiento de las respuestas a las dos afirmaciones fue TA y PA con el enfoque.

Comportamiento de las respuestas de la muestra ¿por qué pregunta?

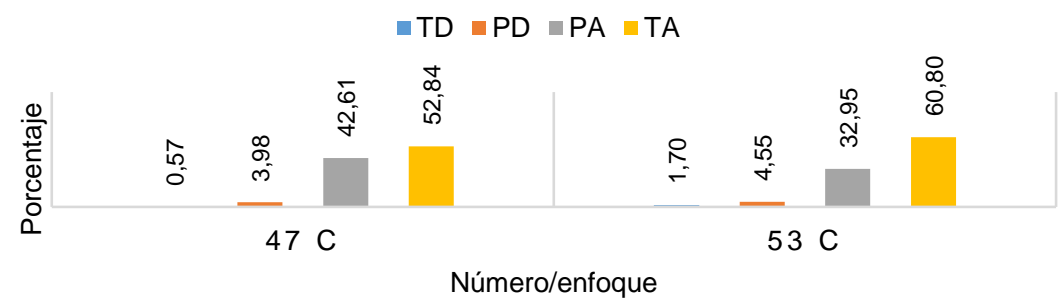

Fig. 9: Agrupación de las afirmaciones y el comportamiento de las respuestas que componen la dimensión ¿Por qué pregunta? C (enfoque constructivista del ítem) y T (enfoque tradicional del ítem).

Los profesores preguntan porque consideran que esto tiene un rol mediador entre lo que el estudiante sabe y lo que desea aprender, además valoran los momentos en los cuales sus estudiantes cuestionan lo que les enseñan. Este resultado sin embargo es contrario a lo presentado por Forbes y Davis (2010) en el cual hacen énfasis en que los profesores no se sienten bien con preguntas en las cuales ellos presentan incertidumbre para responder. El carácter epistemológico de esta dimensión da una gran relevancia a las respuestas provenientes de estas afirmaciones, o sea, no es menor pensar que de un total de 184 profesores, más del $90 \%$ estén de acuerdo o parcialmente de acuerdo con estas afirmaciones. Como lo menciona Degener y Berne, (2017) aunque es difícil capacitar al profesor en el cambio acerca de las preguntas, al hacerlo se eleva la naturaleza de las discusiones en el aula y en consecuencia mejora la mediación y el desarrollo del pensamiento complejo.

\section{Uso de las preguntas}

\section{d. Dimensión “¿Cuándo pregunta en profesor?}

La dimensión ¿Cuándo pregunta el profesor? está compuesta de 5 afirmaciones. Siendo 3 con enfoque constructivista, perteneciendo la primera al G2, la segunda al G3 y la tercera al G1, y 2 con enfoque tradicional, 
categorizadas en el G2 (Fig.10). El comportamiento de las respuestas a las afirmaciones de enfoque constructivista y tradicional fue mayormente TA y PA.

Comportamiento de las respuestas de la muestra ¿cuando pregunta?

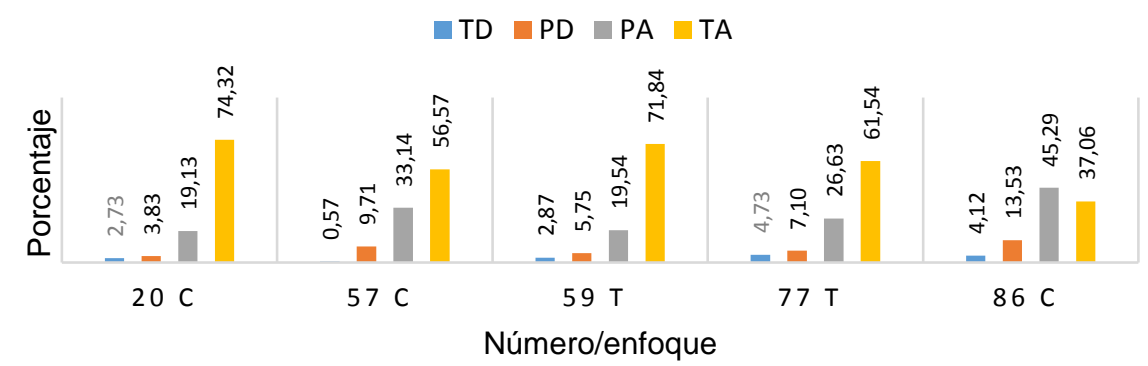

Fig. 10. Agrupación de las afirmaciones y el comportamiento de las respuestas que componen la dimensión ¿Cuándo pregunta? C (enfoque constructivista del ítem) y T (enfoque tradicional del ítem).

El profesor considera útil preguntar a sus estudiantes al término de la clase qué han aprendido, aunque no lo considera importante, lo que es contradictorio. Este hallazgo podría explicarse debido a que los profesores en Chile se les requiere marcadamente la realización del cierre de la clase, haciendo así énfasis en su utilidad, sin embargo, poco es el énfasis en el valor metacongitivo del momento y sus preguntas, lo que es contrario a la idea proveniente de las investigaciones en las cuales se hace énfasis en el uso de las preguntas, partiendo del nivel de pensamiento de orden inferior, avanzando al nivel de pensamiento de orden superior (Degener y Berne., 2017; Roca, et al., 2013) . También considera eficaz la resolución de guías con preguntas abiertas a través de grupos de trabajo y está de acuerdo en que se debe partir la clase con una pregunta que problematice lo enseñado en la clase anterior, lo que está de acuerdo con Nawani, et. al. (2018). Sin embargo estos autores consideran que sus preguntas con enfoque especifico y desafiante involucran significativamente a los estudiantes, lo que es contrario a los resultados identificados en esta investigación en donde los profesores consideran que sin actividad experimental no hay desarrollo del pensamiento científico, lo que indica una comprensión del pensamiento científico centrado exclusivamente en el método y no en una metodología de la ciencia. En cuanto a la afirmación acerca de la importancia de partir la clase con una pregunta que problematice lo enseñado en la clase anterior se muestra una tendencia constructivista y coinciden con lo mencionado por los autores mencionados donde identifican que las preguntas pueden ser usadas como conectores en la enseñanza y la sistematización de los nuevos conocimientos.

\section{e. Dimensión ¿Para qué pregunta el profesor?}

La dimensión ¿Para qué pregunta el profesor? está compuesta de 14 afirmaciones, siendo 8 de ellas de enfoque constructivista, todas pertenecientes al grupo 2, y 6 de enfoque tradicional, siendo 1 del grupo 1 , otra del grupo 3 y las demás del grupo 2. El comportamiento de las respuestas a las afirmaciones con enfoque constructivista fue para 7 de ellas TA y PA, y una tuvo un comportamiento contrario. En las respuestas a las afirmaciones de enfoque tradicional, 4 presentaron un comportamiento TA y PA y dos presentaron TD y PD.

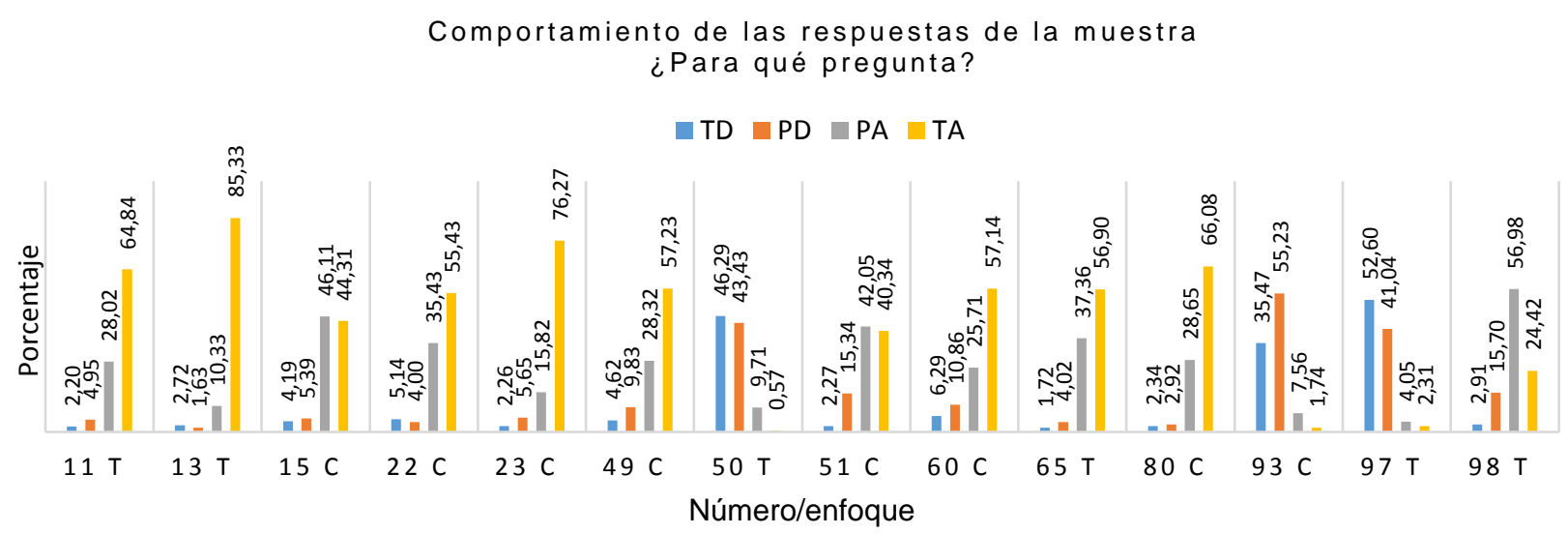

Fig. 11: Agrupación de las afirmaciones y el comportamiento de las respuestas que componen la dimensión ¿Para qué pregunta? C (enfoque constructivista del ítem) y T (enfoque tradicional del ítem).

El profesor pregunta para que los estudiantes puedan articular entre ellos, temas adicionales a los que enseña, para promover el pensamiento divergente en sus estudiantes, porque no está de acuerdo con que 
una pregunta distraiga al estudiante, sin embargo, considera que una pregunta divergente confunde al estudiante, mejora su interés hacia la ciencia, ayuda al estudiante a identificar sus propias dudas, saber lo que realmente sabe, identificar lo que no han aprendido, conocer lo que piensan sus estudiantes, enseñar a través del buen uso del error, este resultado tiene relación con lo presentado por Watson (2017) cuando menciona la relación de la pregunta divergente y la generación de incertidumbre en el aula. Otra de las creencias de los profesores en este estudio es que una pregunta sirve para guiar el pensamiento de su estudiante y centrarlo en el contenido, para saber si sus estudiantes saben el contenido y que se den cuenta en caso que no lo sepan, pero no considera su rol de cómo comunican el contenido. Del mismo modo Watson (2017) indica que los profesores se centran en las preguntas que ellos plantean, por tanto, la dirección que tomará la clase es hacia lo que el profesor considera importante. No obstante Forbes y Davis (2010) en uno de los casos analizados, expresó una insatisfacción con el uso de estas preguntas para obtener las ideas de los estudiantes. El profesor necesita ayudar a su estudiante cuando este no consigue identificar lo que quiere preguntar, para eso reformula la pregunta del estudiante. Esta situación es preocupante ya que según Chin (2007), la interacción en las clases de estos profesores no favorece el desarrollo del pensamiento individual, tampoco el análisis crítico y el pensamiento divergente, en las cuales el estudiantado pudiera mirar problemáticas antiguas desde nuevos enfoques, estrategias y experiencias consolidadas en un paradigma dominante y poco innovador. Más bien sus preguntas se centran en la acumulación de hechos o conocimiento científico canónico, no facilitando la negociación de concepciones alternativas y conceptos preexistentes sobre el tema abordado (Nawani et al., 2018).

\section{f. Dimensión ¿A quién pregunta el profesor?}

La dimensión ¿a quién pregunta el profesor? está compuesta de una afirmación de enfoque constructivista, la cual pertenece al grupo 2 . El comportamiento de las respuestas hacia la afirmación, presenta un predominio en TA y PA.

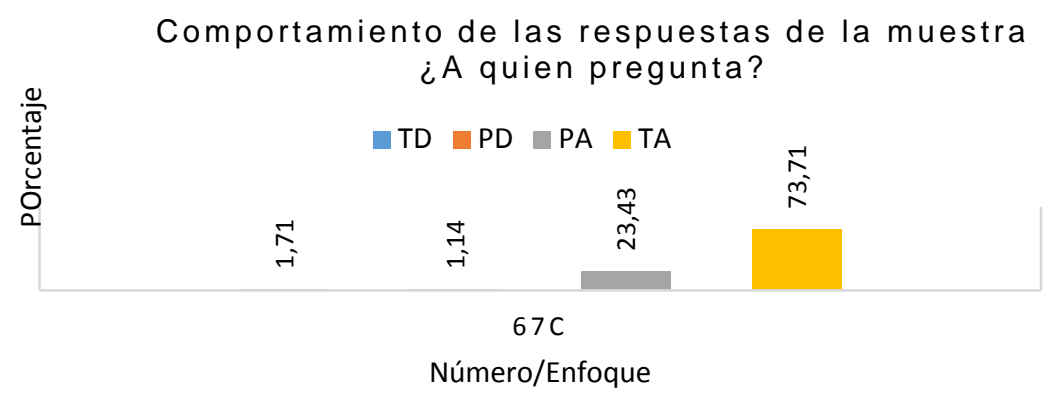

Fig. 12: Agrupación de las afirmaciones y el comportamiento de las respuestas que componen la dimensión ¿A quién pregunta? C (enfoque constructivista del ítem) y T (enfoque tradicional del ítem).

El profesor pregunta a sus estudiantes de manera diferenciada, reconociendo que cada estudiante tiene formas de expresión personales frente a una misma pregunta.

\section{g. Dimensión ¿Cómo se siente el profesor cuando le preguntan?}

La dimensión ¿Cómo se siente el profesor cuando le preguntan? Está compuesta de 2 afirmaciones, las cuales tienen un enfoque tradicional y pertenecen al grupo 2 . El comportamiento de las respuestas fue de TA y PA.

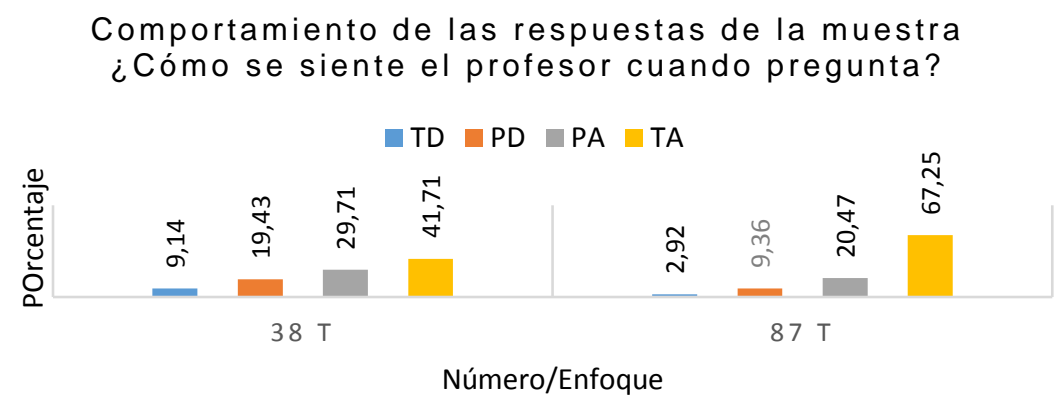

Fig. 13: Agrupación de las afirmaciones y el comportamiento de las respuestas que componen la dimensión ¿Cómo se siente el profesor cuando pregunta? C (enfoque constructivista del ítem) y T (enfoque tradicional del ítem). 
El profesor se siente preocupado si perciben que no conoce una respuesta a una pregunta. Además, se siente ansioso frente a preguntas inesperadas. Este resultado es uno de los que se presentan más claros en la investigación y están de acuerdo con lo obtenido por Watson (2017) acerca de la actitud y emociones experimentadas por el maestro frente a la pregunta inesperada, en este caso la generación de incertidumbre del profesor ocurre debido a estar expuesto al escrutinio y cuestionamiento de su propio conocimiento por parte de sus estudiantes, lo cual es inquietante, perturbador y puede llegar a ser desalentador frente al cambio de roles que este asume. Esto se acentúa si el profesor tiene una percepción propia como guardián del conocimiento. Sin embargo si toma una postura colaboradora, puede ser una excelente oportunidad para aprender junto a sus estudiantes.

\section{h. Dimensión ¿Cómo se sienten los estudiantes cuando pregunta el profesor?}

La dimensión ¿Cómo se sienten los estudiantes cuando pregunta el profesor? está compuesta de 2 afirmaciones, la primera pertenece al grupo 3 y la segunda al grupo 2. El comportamiento de las respuestas tiende a PA y PD.

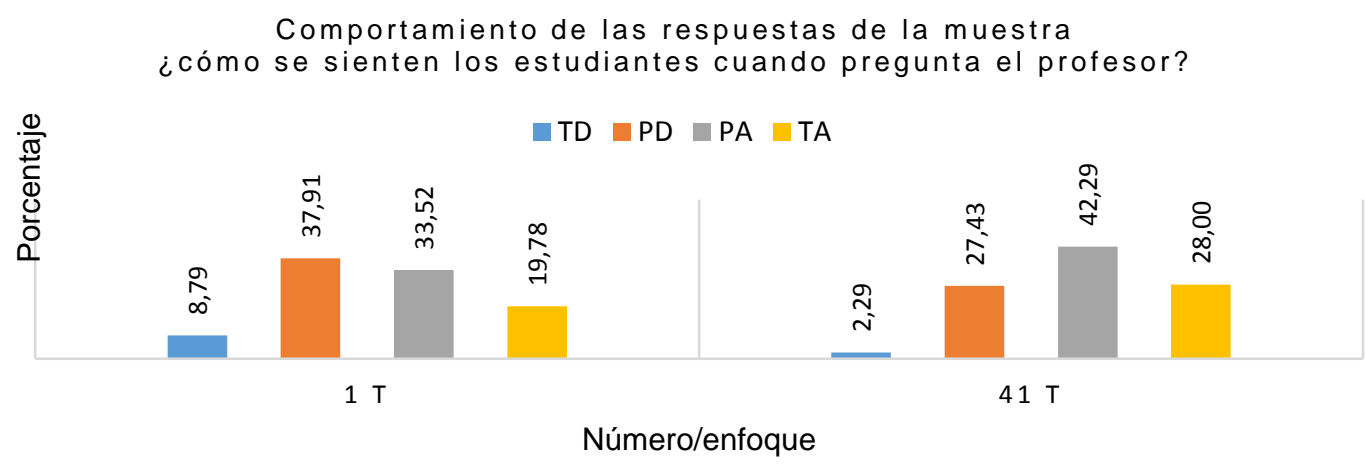

Fig. 14: Agrupación de las afirmaciones y el comportamiento de las respuestas que componen la dimensión ¿Cómo se sienten sus estudiantes cuando pregunta el profesor? C (enfoque constructivista del ítem) y T (enfoque tradicional del ítem).

El profesor cree que sus estudiantes se molestan cuando responde a sus preguntas con otras preguntas y frente a la falta de conocimiento para una respuesta, ellos se sienten agobiados e intentan solapar el déficit respondiendo la pregunta de forma compleja. Esto nos hace reflexionar acerca de la necesidad de desarrollar esta cultura indagatoria en sus clases, o sea, todavía la preocupación del profesor está centrada en sí mismo y no en lo que el estudiante necesita y cómo se siente durante el proceso de indagación para la comprensión. Aun no existen estudios específicos en cómo se sienten los estudiantes en este aspecto.

\section{DISCUSIÓN FINAL}

En lo que se refiere a la formulación de las preguntas, los profesores creen en cuanto al cómo preguntan, que sus preguntas deben ser breves, concisas y generar más de una respuesta. Además usan preguntas abiertas para saber cómo piensan los estudiantes en el aula, pero no las usan en las pruebas, porque son difíciles de evaluar objetivamente y cuando necesitan direccionar un tema en el aula, usan preguntas cerradas. Indicando una priorización del tiempo por sobre lo que consideran ser correcto. Con relación a lo qué preguntan consideran que no deben usar temas dilemas éticos y tampoco problemas inherentes al colegio. Preguntan acerca de contenidos todavía no enseñados, porque consideran que orientan, pero piensan que perturban a los estudiantes. Finalmente, cuanto al por qué preguntan, valoran el cuestionamiento durante el aula y porque consideran que estas son mediadoras entre lo que se sabe y lo que desea aprender,

Respecto al uso de las preguntas, los profesores piensan sobre al cuándo pregunta que es útil preguntar al final de la clase, aunque no lo consideran importante. También preguntan al inicio de la clase o cuando trabajan resolviendo guías en grupo con preguntas abiertas y cuando se requiere desarrollar pensamiento científico en actividades prácticas (experimentales). En lo que concierne al para qué preguntan los profesores aluden a su uso como guía del pensamiento, además de aumentar el interés, promover el pensamiento divergente, si bien también consideran que confunde. Cuanto al uso estas deben posibilitar la identificación de dudas, respondan lo que realmente saben, identificar lo que han aprendido y lo que no, saber cómo piensan, desarrollar su lenguaje y aprendan de sus errores. Sin embargo, consideran que el exceso de preguntas desorienta a sus estudiantes. En cuanto a quien pregunta es el profesor y además espera respuestas diferentes a una misma pregunta. 
En cuanto al cómo se siente el profesor cuando le preguntan, se identifica una fuerte incertidumbre del profesorado frente a las preguntas inesperada que le realiza el estudiante, esto debido a una visión tradicional de su rol, donde el profesor es el detentor y validador del saber. Sin embargo en lo que se refiere a cómo se sienten sus estudiantes se muestran creencias relacionadas con el agobio y la inseguridad en sus estudiantes, posiblemente debido a que provienen de una cultura de la "respuesta correcta" y con menos frecuencia, a situaciones que les ofrezcan oportunidades para aprender desde el error. Es relevante destacar que las creencias identificadas, en su mayoría están centradas en el profesor y poca es la preocupación por los estudiantes.

Si bien las clases de ciencias en la Región Metropolitana de Santiago de Chile son consideradas tradicionales y con fuertes tendencias a la memorización, en este estudio se pudo identificar que las creencias de los profesores, en lo que se refiere a las preguntas y su uso, tienden a un cambio de paradigma, es decir, están marcadamente, "en tránsito" hacia creencias más bien catalizadoras en el aula. Aun así, no indica que esto se aplique en las clases de ciencias a la brevedad, sin embargo podría indicar que ellos ya identifican la necesidad del cambio. Por lo tanto, el transformar el aula de ciencias en espacios donde se pueda desarrollar el pensamiento científico, crítico y autorregulado, a través de la promoción y el uso de buenas preguntas en la clase requiere generar espacios de reflexión y problematización de la práctica donde la interacción entre pares y su consecuente formación profesional en didáctica de las ciencias, son de gran relevancia y podrían tener incidencia en las creencias y también en la acción, permitiendo a ellos identificar incoherencias entre su pensar y su actuar al acortar distancias entre las creencias y la acción didáctica real del aula de ciencias naturales.

\section{CONCLUSIONES}

Concluimos que con relación al instrumento y el comportamiento de los grupos acerca de las afirmaciones, los profesores presentan tendencias de respuestas con un enfoque "tradicional en avance", "en tránsito" y "catalizador". Indicando la convivencia de creencias opuestas y contradictorias en lo que se refiere a las preguntas en la clase de ciencias.

Consideramos que además de las creencias acerca de formulación y uso de las preguntas es necesario relacionar, el enfoque de su enseñanza (en sí mismo o en el estudiante), las políticas públicas, la institución educativa, los padres o las pruebas estandarizadas de aprendizajes, pues son factores que pueden incidir de manera positiva o negativa sobre estas. Por lo tanto, comprender profundamente el proceso de generación de la pregunta y el uso que se le da, es una tarea compleja en la que se necesita avanzar. Su estudio y el desarrollo de múltiples estrategias de cuestionamiento en la interacción en el aula debe ser considerado un componente relevante en el desarrollo profesional de los profesores y de diagnóstico, para acelerar el cambio de prácticas docentes centradas en el profesor hacia aquellas que prioricen el diálogo didáctico, la autonomía del estudiante y su participación en la construcción de significados compartidos en el aula de ciencias.

\section{AGRADECIMIENTOS}

Se agradece el apoyo financiero a la Comisión Nacional de Investigación Científica y Tecnológica a través del proyecto FONDECYT 11150873.

\section{REFERENCIAS}

Abascal, E., y I. Grande, Fundamentos y Técnicas de Investigación Comercial. Edición $13^{\circ}$. Editorial ESIC. Madrid. España (2017)

Bachelard, G., La formación del espíritu científico. Contribución a un psicoanálisis de conocimiento objetivo. Siglo XXI. 20 ed. México (2000)

Benedict-Chambers, A., S. M. Kademian, E. A. Davis y A. S. Palincsar, Guiding students towards sensemaking: teacher questions focused on integrating scientific practices with science content, doi:10.1080/09500693.2017.1366674, International Journal of Science Education, 39(15), 1977-2001. (2017)

Blosser, P. E., How to Ask the Right Questions? NSTA Press, Arlington, USA (2000)

Chen, Y. C., B. Hand y L. Norton-Meier, Teacher Roles of Questioning in Early Elementary Science Classrooms: A Framework Promoting Student Cognitive Complexities in Argumentation, doi:10.1007/s11165-015-9506-6, Research in Science Education, 47(2), 373-405. (2017)

Chin, C., Teacher Questioning in Science Classrooms: Approaches that Stimulate Productive thinking, doi:10.1002/tea.20171, Journal of Research in Science Teaching, 44(6), 815-843 (2007)

Chin, C. y J. Osborne, Students' questions and discursive interaction: Their impact on argumentation during collaborative group discussions in science, doi:10.1002/tea.20385, Journal of Research in Science Teaching, 47(7), 883-908. (2010) 
Cofré, H., y otros cinco autores, La educación científica en Chile: Debilidades de la enseñanza y futuros desafíos de la educación de profesores de ciencias. Estudios Pedagógicos, XXXVI(2), 279-293. (2010)

Colás, C., La Formulación de Preguntas en el Acto Didáctico. Revista interuniversitaria de didáctica, 1, 77-83, (1983)

Contreras, S. A., Qué piensan los profesores sobre sus clases: estudio sobre las creencias curriculares y las creencias de actuación curricular. Formación universitaria, 1(3), 3-11 (2008)

Couso, D., De la moda de "aprender indagando" a la indagación para modelizar: una reflexión crítica. Paper presented at the XXVI Encuentro de Didáctica de las Ciencias Experimentales., Huelva (Andalucía), España. (2014)

Cutrera, G. y S. Stipcich, Enseñanza y Estrategias Discursivas. Principales Aportes desde la Investigación Educativa. Revista Iberoamericana de Producción Académica y Gestión Educativa, 4 (2016)

Degener, S., y J. Berne, Complex Questions Promote Complex Thinking. Reading Teacher, 70(5), 595-599 (2017)

Eliasson, N., H. Sørensen y K. Göran, Teacher-student interaction in contemporary science classrooms: is participation still a question of gender?, DOI: 10.1080/09500693.2016.1213457, International Journal of Science Education, 38:10, 1655-1672 (2016)

Eliasson, N., K. Göran y H. Sørensen, The role of questions in the science classroom - how girls and boys respond to teachers' questions, DOI: 10.1080/09500693.2017.1289420, International Journal of Science Education, 39:4, 433-452 (2017)

Ernst-Slavit, G., y K. L. Pratt, Teacher questions: Learning the discourse of science in a linguistically diverse elementary classroom, doi.org/10.1016/j.linged.2017.05.005, Linguistics and Education, 40, 1-10. (2017)

Ferrés, C., El Reto de Plantear Preguntas Científicas Investigables, Revista Eureka sobre Enseñanza y Divulgación de las Ciencias, 14 (2), 410-426 (2017)

Forbes, C. T., y E. A. Davis, Beginning elementary teachers' beliefs about the use of anchoring questions in science: A longitudinal study, doi: 10.1002/sce.20370, Science Education, 94(2), 365-387 (2010)

Graesser, A., t otros cuatro autores, Inferencias y Preguntas En la Comprensión de Textos Científicos. Tarbiya. Revista de Investigación e Innovación Educativa del Instituto Universitario de Ciencias de la Educación, 103-128 (2005)

Joglar, C., M. Navarro y S. P. R. Rojas, Construct validity in a scale of beliefs of science teachers regarding questions in the classroom. Paper presented at the Electronic Proceedings of the ESERA 2017 Conference. Research, Practice and Collaboration in Science Education, Dublin, Ireland. (2018)

Joglar, C., y S.P. Rojas, Overcoming Obstacles to the Formulation and Use of Questions in the Science Classroom: Analysis from a Teacher Reflection Workshop. DOI: 10.1007/s11165-019-9857-5, Research in Science Education. (En prensa) 1-15. (2019)

Labarrere, A., La solución de problemas, eje del desarrollo del pensamiento y las competencias de pensamiento científico matemáticas y ciencias experimentales. In M. Quintanilla (Ed.), Las competencias de pensamiento científico desde" las voces" del aula: historia de un proyecto de formación continua de docentes basado en la investigación en didáctica de las ciencias. (Vol. 1, pp. 47-82). Santiago de Chile: Bellaterra. (2012)

Lee, M., Productive questions: Tools for Supporting Constructivist Learning. Science and Children, 36(8), 24-27 (1999)

Rojas, R.S.P., y C. Joglar, Small research and asking questions. a study case in initial teacher training in elementary education. Proceedings of INTED2019 Conference, Valencia, Spain. 8884-8889. DOI: 10.21125/inted.2019.2214 (2019)

Machado, V. y L. Sasseron, As Perguntas em Aulas Investigativas de Ciências: a Construção Teórica de Categorias. Revista Brasileira de Pesquisa em Educação em Ciências, 12 (2), 29-44 (2012)

Malvaez, O., C. Joglar y M. Quintanilla, Elaboración de preguntas de los estudiantes para promover la metacognición en el aprendizaje activo en ciencias. IX Encontro Nacional de Pesquisa em Educação em Ciências. 1-8, Águas de Lindóia, Brasil (2013)

Márquez, C. y M. Roca, Plantear Preguntas: Un Punto de Partida para Aprender Ciencias. Revista Educación y Pedagogía, 18(45), 63-71 (2006)

Martín, E., Profesorado competente para formar alumnado competente: el reto del cambio docente. In J. I. Pozo y M. Pérez (Eds.), Psicología del aprendizaje universitario: la formación de competencias. Ediciones Morata, Madrid, España (2009)

Mazzitelli, C.; C. Maturano y A. Macías, Análisis de las Preguntas que Formulan los Alumnos a Partir de la Lectura de un Texto de Ciencias. Revista Electrónica de Enseñanza de las Ciencias, 8(1), 45-57 (2009)

Monteiro, R., J. Carrillo, y S. Aguaded, Guiones de acción de un profesor novel de ciencias a partir de la modelización de la enseñanza. Enseñanza de las Ciencias, 27(1), 077-88. (2009)

Mortimer, E. F., y P. Scott, Meaning Making in Secondary Science Classrooms. UK: McGraw-Hill Education. (2003)

Nawani, J., y cuatro autores, Teachers' Use of Focus Questions in German Biology Classrooms: a Video-based Naturalistic Study. doi:10.1007/s10763-017-9837-z, International Journal of Science and Mathematics Education, 16(8), $1431-1451$. (2018) 
OCDE., Results: Excellence and Equity in Education, PISA 2015 (2016)

Osborne, J., The Role of Argument: Learning How to Learn in School Science. In B. J. Fraser, K. Tobin, y C. J. McRobbie (Eds.), Second International Handbook of Science Education (pp. 933-949). Dordrecht: Springer Netherlands. (2012)

Pajares, M.F., Teachers' Beliefs and educational Research: Cleaning up a Messy Construct. Review of Educational Research, 62(3), 307-362 (1992)

Pappa, E. y G. Tsaparlis, Evaluation of questions in general chemistry textbooks according to the form of the questions and the Question-Answer Relationship (QAR): the case of intra- and intermolecular chemical bonding. Chemistry Education Research and Practice, 12(2), 262-270 (2011)

Porlán, R., y otros cinco autores, El cambio del profesorado de Ciencias II: Itinerarios de progresión y obstáculos en estudiantes de Magisterio. Enseñanza de las Ciencias. Revista de Investigación y Experiencias Didácticas, 29 (3), $353-$ 370. (2011)

Redfield, D. L. y E. W. A. Rousseau, Meta-Analysis of Experimental Research on Teacher Questioning Behavior, doi:10.2307/1170197, Review of Educational Research, 51(2), 237-245 (1981)

Roca, M., C. Márquez, y P. N. Sanmartí, Las Preguntas de los Alumnos: Una Propuesta de Análisis. Enseñanza de las Ciencias, 31(1), 95-114 (2013)

Sanmartí, N., y I. Marchán, ¿Cómo Elaborar una Prueba de Evaluación Escrita? Alambique Didáctica de las Ciencias Experimentales, 78, 1-10 (2014)

Sanmartí, N., y C. Márquez, Enseñar a Plantear Preguntas Investigables. Alambique (enero-febrero-marzo), 27-36 (2012)

Silvestri, A., La Formulación de Preguntas para la Comprensión de Textos: Estudio Experimental, doi:10.4067/s071809342006000300008, Revista signos, 39 (62), 493-510 (2006)

Sun, Y., M. Correa, A. Zapata y D. Carrasco, Resultados: Qué Dice la Evaluación Docente Acerca de la Enseñanza en Chile (F. d. C. Sociales, Trans.). En J. Manzi, R. González, y Y. C. Sun (Eds.), La Evaluación Docente en Chile, 1-262. MIDEUC, Santiago, Chile (2011)

Vázquez-Bernal, B., R. Jiménez-Perez y V. M. Jiménez, ¿El Tiempo garantiza el cambio en el profesorado? Estudio de un Caso centrado en la evaluación de aprendizajes, Revista Electrónica Interuniversitaria de Formación del Profesorado, 19(2), 139-154. (2016)

Watson, E., Unexpected Questions: Reflecting on the Teacher's Experience of Responding in Class, doi:10.1353/hsj.2017.0015101, High School Journal, (1), 49-61 (2017)

Wertsch, J. V., Vygotsky y la Formación Social de la Mente. Paidós, Barcelona, España (1988) 